\title{
Acquisition footprints extracting from raw data can be estimated by the azimuth and offset Entropy of the geometry
}

\author{
DengKe $\mathrm{He}^{*}$ \\ China University of Mining \& Technology (Beijing) \\ Beijing, China \\ He_dengke@126.com
}

\begin{abstract}
- to be differently using the amplitude section from stack volume, the acquisition footprint can be represented by energy distribution of the raw seismic in the paper. Furthermore, the paper discussed the distributions of the offset entropy and azimuth entropy of the geometry, described the similarity between the offset entropy or azimuth entropy and the acquisition footprints as well. According to these similarities, the offset and azimuth entropy can guide to not only design and evaluate quantitatively the acquisition geometry, but also estimate the acquisition footprints.
\end{abstract}

Keywords-aquisition footprints; raw seismic; offset entropy; azimuth entropy;

\section{INTRODUCTION}

The acquisition footprint is an important factor contaminated the processing data volume of the seismic prospecting. Various reasons from the acquisition progress and signal processing may produce an acquisition footprint, but the surface acquisition geometry, including the source array, the receiver array and the relationship between the both above, could be distinguished ${ }^{[1]}$. Many papers have reported that the acquisition footprints could be detected on the stack volumes and have proposed many corresponding removing methods. Actually, the acquisition footprints can be seen from the raw seismic data. In this paper, we study the acquisition footprints abstracted from the raw data and compare them with the azimuth entropy and offset entropy, which were introduced by Peng et al (2010).

\section{ACQUISITION FOOTPRINTS IN THE RAW SEISMIC DATA EXAMPLES}

The geometry array discussed in the paper, from a seismic prospecting project of Henan province of China, consists of a source line with four sources by 10 meters interval and eight receiver lines with 72 receiver stations per line (seeing from the top of Fig.1). The source points are at the start of the receiver lines. The minimum offset is 5 meters. The receiver points separate along the line by 20 meters, the receiver lines space is 40 meters apart. Rolling distances along the receiver line direction and its perpendicular direction are 60 meters and 80 meters, respectively. The planning fold is 24 on a bin with 5 meters multiplied 10 meters, and the actual maximum fold is 41 due to the geometry altering. The detail of the fold distribution describes on the bottom of Fig.1.

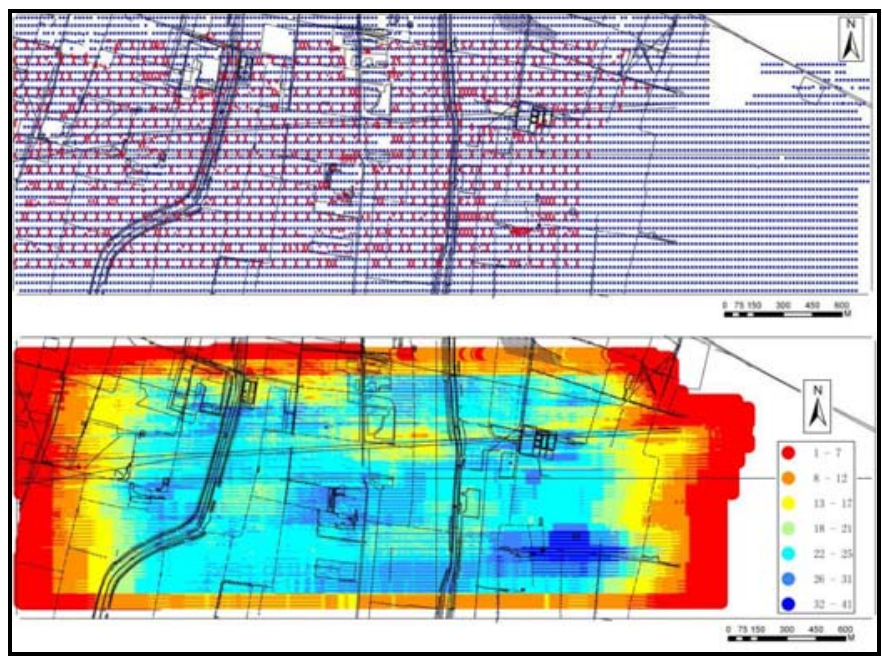

Figure 1 Geometry (top, red: source, blue: receiver) and its CDP fold map (bottom)

The acquisition footprints represent the no uniform distribution of seismic amplitudes about the stack volumes,

especially on the horizontal section of the object events. They could make interpreters misinterpreting usually with some 
geological structures. The acquisition footprint seeing from stack volumes is a focus of processing and interpreting consequently. However, there are also obvious footprints on the raw data. We adopt the maximum differences of the average energy measured from all traces with a given window on the CDP bin to represent the acquisition footprints. The average energy distributions are respectively calculated from the surface wave area and the valid signal area, shown in the Figure 2.

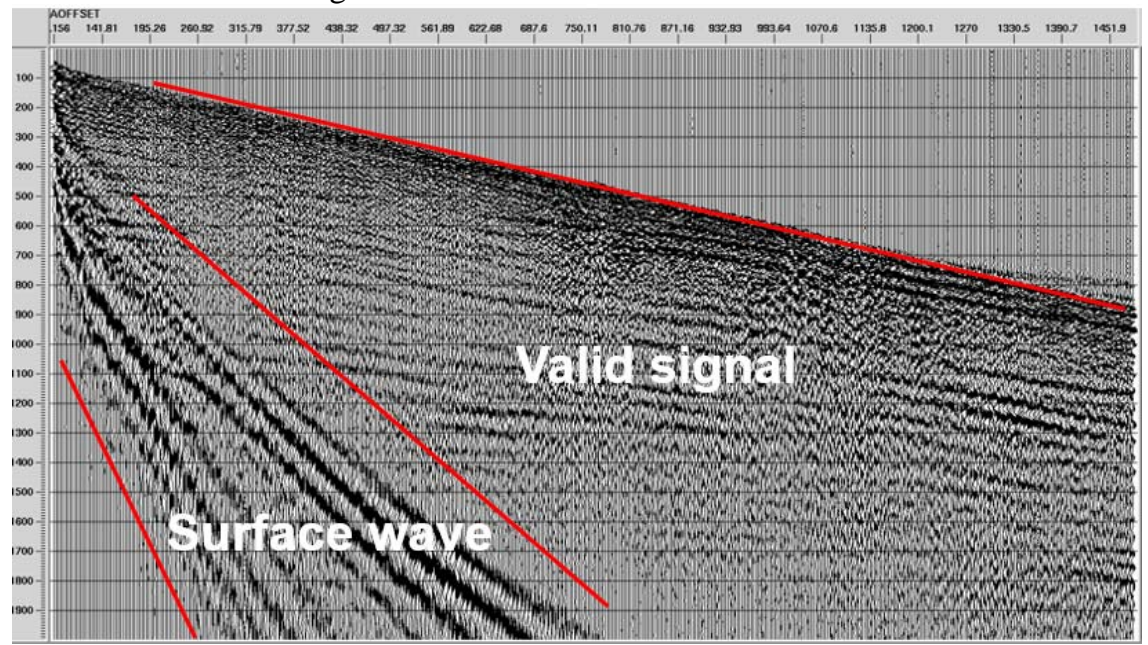

Figure $2 \mathrm{~A}$ raw source gather with offset to specify the surfave wave window and the valid signal window

The surface factors about the source points or the receiver points will lead to acquisition footprints, meanwhile, the AVO factors or AVAz factors will also lead to acquisition footprints. The latter is closely related to the features of the acquisition geometry. Therefore, the acquisition footprints caused by the geometry features is the major topic for distinguishing and removing acquisition footprint to some extent.

It is customary to distinguish and describe the acquisition footprints in the stack sections. However, the acquisition footprints can be found in the raw seismic data. The theoretical hypotheses of the NMO stack or migration stack is that the energy factor of each trace with different offset is equal. Actually, the factors with different offsets are different, which will cause the acquisition footprints. To some extent, the difference between the max energy and the min energy can represent the acquisition footprint. Similarly, the maximum differences of the average energy measured from all raw traces with a given window on the CDP bin can describe the acquisition footprints.
Seeing from the Figure 3, the energy distributions of the surface wave window are shown in the top frame and the energy distributions of the valid signal window are shown in the bottom frame. The energy differences from the surface wave window and the valid signal window spread regularly by 80 meters along receiver lines direction (from west to east) and 60 meters along perpendicular receiver lines direction (from south to north), which are in accord with the rolling distances of the geometry pattern on the both directions, shown in the top of the Figure 1. The regular patterns of the energy differences from both areas are the acquisition footprints because their motion regularities agree with the geometry and the energies are the statistical from corresponding windows not any special event. It is the geometry no uniformity about the offset or azimuth distribution not the un-continuity of a special stratum to generate the footprints.

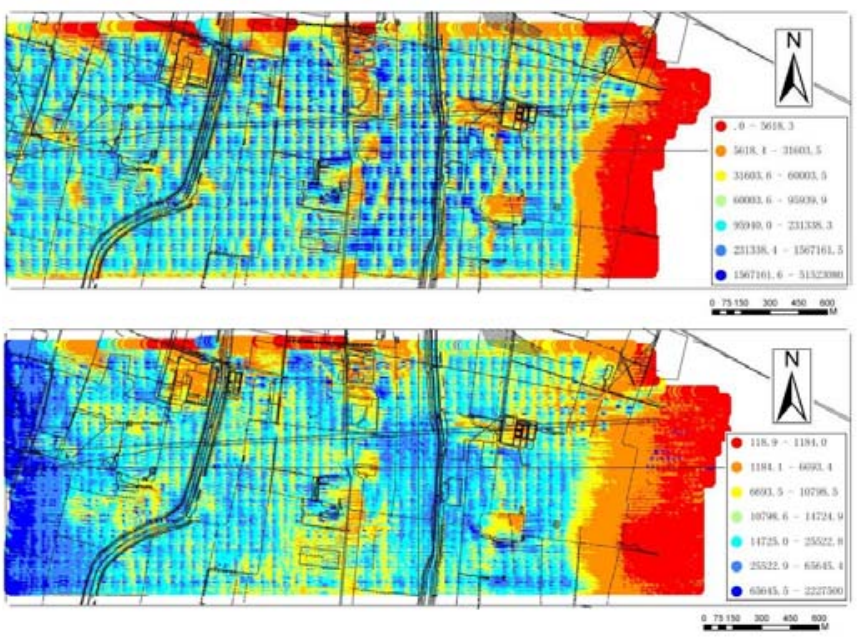

Figure 3 The maximum differences of average energy of surface wave window $(\mathrm{T})$ and valid signal window (B) 


\section{OFFSET ENTROPY AND AZIMUTH}

The offset entropy and azimuth entropy are introduced by Peng et al. (2010) according to Shannon (1948) to quantitatively evaluate the offset and azimuth uniform of a given acquisition geometry ${ }^{[2,3]}$. Shannon (1948) defined the signal entropy as following ${ }^{[4]}$

$$
H=-Q \sum_{n=1}^{N} p_{n} \log _{2} p_{n}
$$

Where $Q$ is a positive is constant, $P_{n}$ is the probability of the $n$ kind of realization, and $N$ is the total number. In the same way, Peng et al (2010) defined the offset or azimuth entropy as following

$$
B=-\sum_{m=1}^{M} p_{m} \log _{2} p_{m}
$$

If the fold of the calculating bin is $N$, the total number of the offset sections or the azimuth sections is $M$, then the probability $P_{m}$ of the $m^{\text {th }}$ section with $k$ traces is equal to $k / N$. The offset section or azimuth section is divided by some a quantity which is arbitrarily selected according to the request of the exploration resolution. The maximum entropy $B$ is a constant. The smaller the difference between the maximum and the real entropy of a bin, the more uniform the offset distribution or azimuth distribution of the bin is.

In the paper, the offset range splits into 32 sections with 50 meters interval, and the azimuth range splits into 12 sections with 30 degrees interval. The ideal offset entropy and azimuth entropy are equal to 5.0 and 3.5850, respectively. Both distributions of the research area are shown in the Figure 4, the top frame shown the offset entropy and the bottom one shown the azimuthal entropy.

The distributions are regular with the acquisition geometry. Simultaneously, the distributions are regular with the energy distributions of the surface wave area and the valid signal area, seeing from the Figure 3 and Figure 4. Especially, the surface wave energy varies closely with the azimuth entropy. Firstly, the high value areas from the azimuth entropy largely in accord with the low differences of the surface wave energy. Secondly, the little triangle patterns from the azimuth entropy move with these notches of the difference patterns of the surface wave energy. The similarities between the entropy and footprints indicate that the entropy can estimate the acquisition footprints.

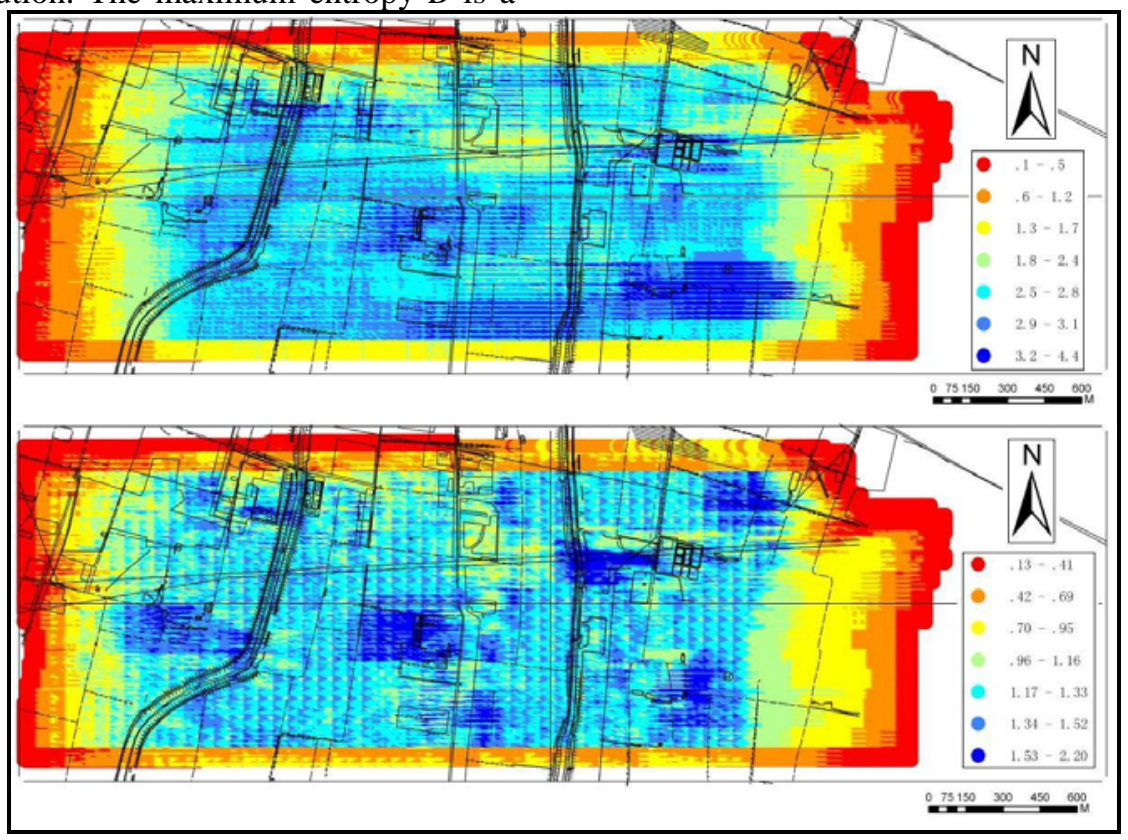

Figure 4 Offset Entropy (top, ideal value=5.0) and Azimuth Entropy (bottom, ideal value=3.5850)

\section{CONCLUSIONS}

Acquisition footprint represented by regular signal on the stack volume is drawing more attention due to disturbing seismic interpretation badly. These examples and removing methods can be referenced many other papers. Actually, acquisition footprint can be exhibit by raw seismic data. In the paper, the footprints can be represented by the maximum differences of energy calculated in a given window, such as in surface wave window or valid signal window, about each CDP bin.
Otherwise, both azimuth entropy and offset entropy can guide to not only design and evaluate quantitatively the acquisition geometry, but also represent and estimate the acquisition footprints.

\section{ACKNOWLEDGEMENTS}

The author thanks these following projects for financial supporting this research, including the Coal United Project of National Natural Science Foundation (U1261203), China Geological Survey Project (1212011220798), National Science and Technology Major Project (2011ZX05035-004001HZ). 


\section{REFERENCES}

[1] Marfurt K. J., Scheet R. M., Sharp J. A., et al., "Suppression of the acquisition footprint for seismic sequence attribute mapping," GEOPHYSICS, vol.63, pp. 1024-1035, May 1998.
[2] Peng S. P., He D. K., Gou J. W., et al., "Quantitative evaluating offset and azimuth uniformity based on entropy," The EAGE Developments in Land Seismic Acquisition for Exploration, May 2010.

[3] Shannon, C. E., "A mathematical theory of communication," The Bell System Technical Journal, vol.27, pp. 379-423, July 1949

[4] Sheriff R. E. "Encyclopedic dictionary of applied geophysics,"SEG, January 2002. 